\title{
Network Pharmacology Approach to Uncover the Mechanism Governing the Effect of Radix Achyranthis Bidentatae on Osteoarthritis
}

Laigen Zhang ${ }^{\dagger}$, Xiaoqing Shi ${ }^{\dagger}$, Zhengquan Huang, Jun Mao, Wei Mei, Liang Ding, Li Zhang, Runlin Xing and Peimin Wang ${ }^{*}$

\begin{abstract}
Background: This study used a network pharmacology approach to elucidate the molecular mechanism governing the effect of Radix Achyranthis Bidentatae (RAB) on osteoarthritis (OA).

Methods: Based on oral bioavailability and drug-likeness, the main active components of RAB were screened via the Traditional Chinese Medicine Systems Pharmacology platform. The GeneCard, OMIM, PharmGkb, Therapeutic Targets database, and DrugBank database were used to establish a database of osteoarthritis targets. The interactive active network map of "ingredient-target" was constructed with Cytoscape software (Version 3.7.1). The proteinprotein interaction network was constructed with the STRING database, and the related protein interaction relationship was analysed. GO biological function analysis and KEGG enrichment analysis for core targets were performed. Finally, docking of the active components with the core target was carried out.

Results: Sixteen active components of RAB were obtained, and 63 potential targets for OA were identified. Network analysis results indicate that these targets are primarily involved in regulating biological processes, such as cell metabolism, apoptosis, and cell proliferation. Pathways involved in the treatment of osteoarthritis include virusrelated signalling pathways, apoptosis signalling pathways, IL-17 signalling pathways, and PI3K/AKT signalling pathways.

Conclusion: RAB has the characteristics of being multi-system, multi-component and multi-target. Possible mechanisms of action for RAB include regulating the immune and inflammatory responses, reducing chondrocyte apoptosis, and protecting the joint synovial membrane and cartilage to control disease development. The active ingredients in RAB, such as sterols and flavonoids, exhibit strong potential as candidate drugs for the treatment of osteoarthritis.
\end{abstract}

Keywords: Network pharmacology, Mechanism prediction, Radix Achyranthis Bidentatae

\footnotetext{
* Correspondence: drwpm@163.com

†Laigen Zhang and Xiaoqing Shi contributed equally to this work. Department of Orthopaedics and Traumatology, The First Affiliated Hospital of Nanjing University of Chinese Medicine, Jiangsu Provincial Hospital of Traditional Chinese Medicine, No 155, Hanzhong Road, Nanjing 210029, Jiangsu Province, China
}

(c) The Author(s). 2020 Open Access This article is licensed under a Creative Commons Attribution 4.0 International License, which permits use, sharing, adaptation, distribution and reproduction in any medium or format, as long as you give appropriate credit to the original author(s) and the source, provide a link to the Creative Commons licence, and indicate if changes were made. The images or other third party material in this article are included in the article's Creative Commons licence, unless indicated otherwise in a credit line to the material. If material is not included in the article's Creative Commons licence and your intended use is not permitted by statutory regulation or exceeds the permitted use, you will need to obtain permission directly from the copyright holder. To view a copy of this licence, visit http://creativecommons.org/licenses/by/4.0/. The Creative Commons Public Domain Dedication waiver (http://creativecommons.org/publicdomain/zero/1.0/) applies to the data made available in this article, unless otherwise stated in a credit line to the data. 


\section{Background}

Osteoarthritis (OA) is a common chronic disease with a significant impact on human health. With the ageing of the general population, the incidence of OA has been increasing annually [1]. In 2014, $13 \%$ of the elderly were diagnosed with OA according to population-based healthcare data from England and Sweden [2]. In medical findings concerning people over 55, approximately $25 \%$ of the population reports at least one knee pain attack every year, which is likely to reflect the potential of OA [3]. Pain and dysfunction significantly reduce patients' quality of life and cause serious social and economic burdens. In the statistics regarding years living with disability, OA ranks first among non-infectious diseases [4].

Chinese herbal medicine (CHM) has a long history and unique features for the treatment of OA; $\mathrm{CHM}$ is observed to be effective in the improvement of clinical symptoms, such as pain and stiffness, with few side effects and lower costs than Western medicine [5-8]. Pan and Huang [9, 10] found that Radix Achyranthis Bidentatae (RAB) is employed most frequently in the treatment of knee osteoarthritis ranked first among other herbs.

The source of Achyranthes bidentata is the root and rhizome of Achyranthes aspera L., which is a traditional Chinese medicinal plant in China [11]. Achyranthes bidentata is widely distributed in tropical and subtropical regions. According to the ancient books of traditional Chinese medicine, the main effects of RAB include nourishing the liver and kidney, strengthening bones and muscles, and invigorating circulation. Modern medicine's research on Achyranthes bidentata primarily focuses on the biological effects on the immune system, nervous system, and bone metabolism, as well as antitumour, antioxidation, and joint-protection properties [12, 13]. Polysaccharides, polypeptides, alkaloids, triterpenoid saponins, organic acids, ketosteroids and various trace elements are known as the primary classes of bioactive ingredients [12].

Achyranthes bidentata Bl. saponins (ABS) can not only promote osteogenic differentiation of bone marrow mesenchymal stem cells by activating the ERK signalling pathway but also protect chondrocytes by inhibiting the activation of $\mathrm{NF}-\mathrm{kB}$ in rat cartilage induced by $\mathrm{IL}-1 \mathrm{~B}$, thereby inhibiting IL-1B-induced apoptosis [14, 15]. Achyranthes bidentata polysaccharides (ABPs) promote the proliferation of chondrocytes and inhibit the production of osteoclasts, possibly by activating the $\mathrm{Wnt} / \beta$-catenin signalling pathway and inhibiting RANKL signalling [16, 17]. In addition, other studies have shown that Achyranthes bidentata alcohol promotes the proliferation of osteoblasts in a dose-dependent manner, possibly by activating the ERK signal transduction pathway to stimulate osteoblast differentiation [18].

The pharmacological study of $\mathrm{ARB}$ and the mechanism of its treatment of OA have been investigated by a number of studies. However, the drug compositions of ARB are complex, and the specific mechanism of its treatment of OA still needs to be fully elucidated. CHM treatment of diseases has the characteristics of "many-to-many-to-many", that is, the complex process of multiple targets to multiple channels to multiple genes. At present, it remains difficult to explore the potential molecular mechanism of $\mathrm{CHM}$ because of the diversity of its components and the complexity of its interactions with the human body. Traditional single-agent or monomer studies were unable to fully explain the specific mechanism of CHM treatment of certain diseases. Based on the development of systems biology and bioinformatics, modern technology has entered the era of omics and big data. Researchers have attempted to explore the correlation between diseases and drugs from the perspective of network biology. In 2007, Chinese scholar Li Shao et al reported the network regulation effects of the TCM syndrome biomolecular network and proposed a biomolecular network to study the active compounds of Chinese medicine [19]. Then, based on the theory of systems biology, Hopkins first proposed the term "network pharmacology." Hopkins explained the relationship between drugs and the body from the perspective of improving or restoring the balance of biological networks and built a "drug-targeted disease" network [19].

Network pharmacology is based on the "disease-genetarget-drug" interaction network, which systematically and comprehensively characterizes the intervention and influence of drugs on the disease network, thereby elucidating the synergistic effects of drugs on the human body. The booming development of bioinformatics provides the possibility to clarify the mechanism of TCM for the treatment of diseases.

Based on the network pharmacology approach, this study focuses on the mechanism governing the effect of $\mathrm{RAB}$ on $\mathrm{OA}$ at the molecular level, attempts to elucidate the specific targets and molecular signalling pathways of $\mathrm{RAB}$ acting on $\mathrm{OA}$, and devises new strategies for drug development and clinical applications. A flowchart of this study is depicted in Fig. 1.

\section{Methods}

\section{Compound collection and screening}

Traditional Chinese Medicine Systems Pharmacology (TCMSP) is a unique system pharmacology platform of $\mathrm{CHM}$ that captures the relationships among drugs, targets and diseases [20]. In TCMSP, we retrieved the 


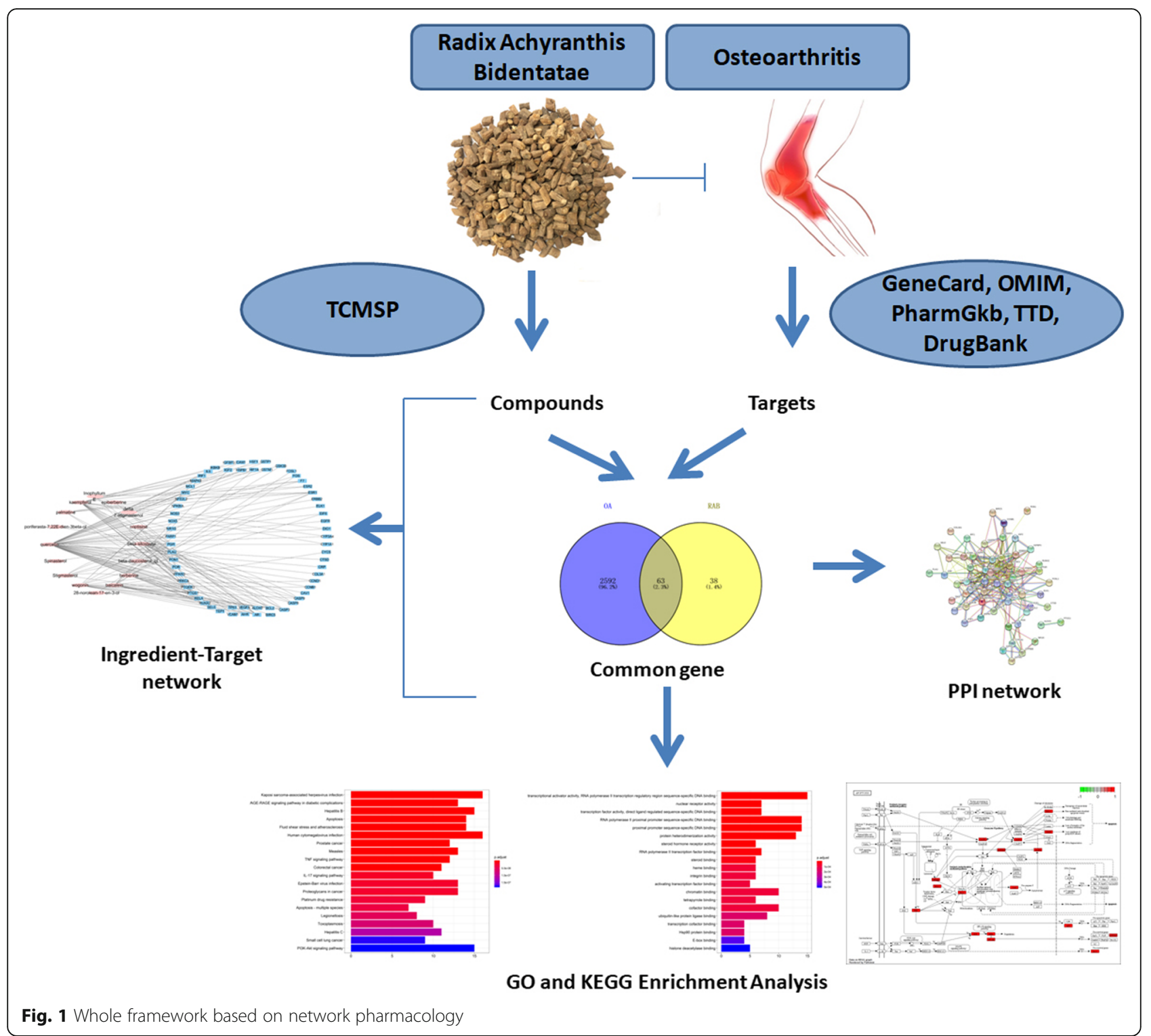

chemical composition information of RAB. The active ingredients of $\mathrm{RAB}$ were obtained from the screening conditions as follows: oral bioavailability $(\mathrm{OB})>30 \%$ and druglikelihood (DL) $>0.18[21,22]$. Then, the potential targets of RAB were collected through the TCMSP platform. With the UniProt database (https://www.uniprot.org/uniprot/), the RAB gene target was obtained.

\section{Target acquisition for drug and disease-related effects}

Using osteoarthritis as a keyword, the OA gene was searched and screened by the following electronic databases: GeneCard database (https://www.genecards.org/), OMIM database (https://www.omim.org/), PharmGkb database (https://www.pharmgkb.org/), Therapeutic Targets database (http://bidd.nus.edu.sg/BIDD-Databases/
TTD/TTD.asp), and DrugBank database (https://www. drugbank.ca). The database search results are combined, and duplicate targets are deleted to obtain all the target targets of OA (the results are shown in Supplementary Table 1). RAB matched the target prediction results of active ingredients with the retrieval results of OArelated targets and selected overlapping targets as the related targets of RAB for the treatment of OA. The Venny 2.1 (http://bioinfogp.cnb.csic.es/tools/venny/ index.html) online tool was used to map the active ingredient target and the disease target of $\mathrm{RAB}$ and to draw Venn diagrams. Then, the intersection targets of $\mathrm{RAB}$ and $\mathrm{OA}$ were collected as the common genes of $\mathrm{RAB}$ in the treatment of $\mathrm{OA}$, which might be the potential target set of RAB in the treatment of OA. 


\section{Construction of drug active ingredients and disease target network}

The screening results of the active components of $\mathrm{RAB}$ and OA targets were imported into Cytoscape [23], and the active components and OA target network of RAB were constructed. The Network Analyzer Apps software was then used to analyse the topology properties of the network, including degree, betweenness centrality, and closeness centrality.

In this experimental study, degree refers to the number of direct neighbours of a node. It is considered that the greater the number of directly connected nodes of a node are, the greater the influence is [24]. Betweenness centrality measures the shortest path between all pairs of nodes through the node $[25,26]$. Closeness centrality is defined as the reciprocal of the average of the shortest path distance between a node and all other nodes in the network, and the larger the value is, the greater the centrality of the node is, indicating that the signal is passed from one node to other nodes faster.

The nodes of the network represented the target or active component, and the edges indicated their interaction. The core architecture of Cytoscape software was the network. Each node was a protein or an active component. The edge between nodes represents the interaction between these biomolecules. The degree of nodes represents the number of nodes connected with one another in the network. A node with a high degree of betweenness centrality and closeness centrality values means that the node plays a highly important role in the network.

\section{Protein-protein interaction (PPI) network construction and core gene screening}

PPI refers to the process in which two or more protein molecules form protein complexes through noncovalent bonds. PPIs and acquired networks are highly important in most biological functions and processes [27]. The STRING database (https://string-db.org/) collected a large number of protein interactions and contains a range of data confidence (low confidence: $<0.4$; medium confidence: 0.4-0.7; high confidence: $>0.7$ ). On this basis, common gene targets of $\mathrm{RAB}$ and $\mathrm{OA}$ were imported into the STRING database. Using the speciesqualified Homo sapiens, a confidence level of 0.7 and hidden disconnected nodes in the network, a protein interaction network analysis was carried out, and the TSV format of the updated results was downloaded. The file was imported into Cytoscape for topology attribute analysis. We then assessed the topological property of nodes in the interaction network by calculating six parameters with the App CytoNCA [28]: "degree centrality (DC)," "betweenness centrality (BC)," "closeness centrality (CC)," "eigenvector centrality (EC)," "network centrality (NC)," and "local average connectivity (LAC)." These six parameters measured the importance of nodes in the network and indicate the nature of the nodes in the network. A node with high DC, BC, CC, EC, NC and LAC values means that it plays a highly important role in the network. Based on the results of topological property analysis of the above PPI, targets above the median were selected as the core targets and screened twice [21]. Based on the above findings, we obtained the core target in the network.

\section{GO and KEGG enrichment analysis}

A variety of bioinformatic analyses and visualization of the results can be achieved using the software $\mathrm{R}$ project. First, the Entrez gene ID of the core target was obtained by RGUI and org. Hs.eg.db, and then the GO function enrichment (Molecular Function, MF, Biological Process, BP, Cellular Component, CC) and KEGG pathway were enriched by RGUI, DOSE and Cluster Profiler. In the programming language, pvalueCutoff $=0.05$ and qvalueCutoff $=0.05$ were set. The results of the analysis selected the top 20 items with the highest enrichment and displayed them in the form of bar graphs. Finally, using RGUI and pathview, the top 20 access maps of the enrichment rankings were downloaded through the KEGG database (KEGG, https://www.kegg.jp/kegg/pathway. html, updated on July 1, 2019). The results were screened repeatedly and manually selected based on relevance.

\section{Acquisition of drug-like components and $\mathrm{OA}$ target crystals}

Through the construction of the target network of the active ingredient of the drug and the disease, the druglike component of the $\mathrm{RAB}$ and the target of $\mathrm{OA}$ were obtained. The mol2 structure of the abovementioned drug-like component, celecoxib (positive control drug), was downloaded from the Chemical Book database (https://www.chemicalbook.com/). The protein crystal structure of the OA-related target was downloaded from the RCSB PDB database (https://www.rcsb.org/).

\section{Docking steps and results evaluation}

The results obtained in 2.6.1 were sequentially imported into Discovery Studio 20163.0 software for preprocessing. First, Prepare Ligands is applied to each drug component, and the parameters are default values and saved as molecular docking ligands. Second, through Prepare Protein, the parameters take the default value, the pre-treated protein structure is obtained, the original ligand is extracted from the target, and the molecule is paired as an acceptor. The receptor active site selects the original ligand extraction position, the docking preference is set to high quality in the LibDock module, the 
conformation method is BEST, parallel processing is true, and other parameters take the default values. The docking results are given by the scoring function LibDock score. The higher the LibDock score is, the higher the activity of the predicted component binding to the target is.

\section{Results}

\section{Screening of active ingredients in RAB}

The TCMSP database was searched using the screening conditions $\mathrm{OB}(>30 \%)$ and DL $(>0.18)$. A total of 20 active ingredients were found in RAB. Twenty active ingredients were manually searched for related targets, and 16 active ingredients were finally selected, as detailed in Supplementary Table 2. Among these ingredients, phytosterols, such as stigmasterols, berberine and other alkaloids, have been proven to have anti-inflammatory, antioxidation, anticancer and other broad pharmacological effects [29-32]. Flavonoids, such as quercetin and baicalein, can eliminate cartilage degeneration, inhibit chondrocyte apoptosis in the knee joint, reduce the oxidative stress response and inhibit the degradation of the extracellular matrix of chondrocytes [33-36].

\section{Construction of the effective active ingredient pool and disease target set}

The targets in the TCMSP database were searched, and a total of 513 targets for the RAB were included. The gene names of the targets were collected using the
UniProt database, null and repetitive targets were deleted, and 101 effective active ingredient targets were obtained. A total of 2655 OA gene targets were obtained by searching and integrating the GeneCard, OMIM, PharmGkb, Therapeutic Targets database, and DrugBank database. A total of 101 compound targets were mapped to 2655 OA target genes to obtain 63 common target genes, as shown in Supplementary Table 3.

\section{Building a "ingredient-target network" network}

Sixteen drug components and sixty-three disease-related target screening results were mapped, chemical components without corresponding targets were removed, and duplicated targets were deleted. The data were imported into Cytoscape to construct an ingredient-target network, as shown in Fig. 2, and the docking results of RAB to the OA protein receptor were shown in Supplementary Table 4. (The octagonal node represents the active pharmaceutical ingredient, such as quercetin; the circular node represents the target of disease action, such as silk mitogen-activated protein kinase 8 (MAPK8).) As shown in Fig. 2, the network includes a total of 79 nodes and 151 sides. Different nodes represent the active constituents of RAB and the target of OA. Figure 2 shows that $\mathrm{RAB}$ can correspond to one or more active ingredients for one target, and multiple targets can correspond to the same active ingredient, suggesting that RAB has multi-component and multi-target characteristics for the treatment of OA.

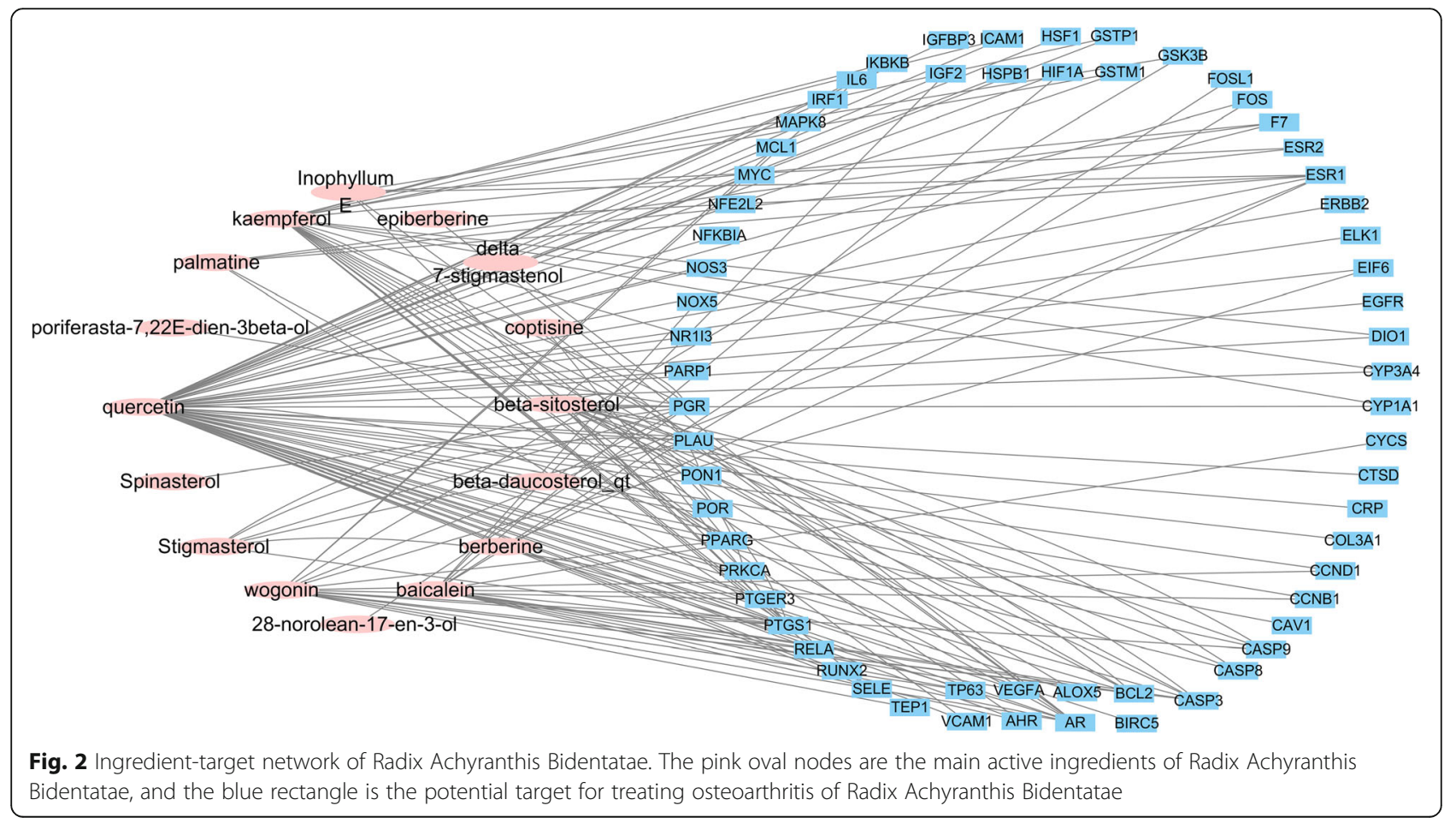




\section{Target protein interaction network analysis}

The common targets related to OA treatment of RAB were imported into the STRING database to obtain their interaction relationship. The interaction network has 58 nodes and 241 edges. TSV data were imported into Cytoscape, and CytoNCA was used to analyse the above PPIs based on DC, BC, CC, EC, NC, and LAC parameters. The thresholds for the first screening were Degree> 6 , Eigenvector $>0.084871295$, LAC $>3.2$, Betweenness $>$ 16.682932, Closeness $>0.243071465$, and Network $>4$, and the results showed a total of 19 nodes and 91 edges. Then, 19 targets were further screened. The second screening threshold was Degree> 14, Eigenvector> 0.17461096, LAC> 5.7894735, Betweenness > 130.68596, Closeness $>0.2626728$, and Network $>8.641908$. The second screening result was 7 nodes and 20 edges (Fig. 3), including MAPK8, IL6, VEGFA, EGFR, MYC, CCND1, and CASP3.

\section{GO and KEGG pathway enrichment analysis}

The MF, BP and CC of 63 core targets were analysed, and the results showed that the key targets of $\mathrm{RAB}$ were highly enriched in 79 GO terms. The biological functions and processes involved, including specific sequence DNA binding, RNA polymerase II proximal promoter sequence-specific DNA binding, transcriptional activator binding, chromatin binding, cofactor binding, nuclear receptor activity, steroid hormone body activity, and nuclear chromatin binding, were closely related to biological processes, such as cell proliferation and apoptosis. The top $20 \mathrm{GO}$ analysis results were screened, with $P<0.05$ serving as the threshold, as shown in Fig. 4 . KEGG enrichment analysis was performed on 63 targets of RAB. The results showed that the targets were enriched in 112 pathways, including viral-related signalling pathways, apoptosis signalling pathways, IL-17 signalling pathways, and PI3K/AKT signalling pathways. These classical signalling pathways play an important role in the occurrence and development of OA. The top 20 KEGG analysis results were screened, with $\mathrm{P}<0.05$ serving as the threshold, as shown in Fig. 5. GO and KEGG analyses suggested that RAB can act on OA through multiple pathways.

\section{Target path analysis}

The KEGG Mapper tool was used to obtain the pathway map of RAB for the treatment of OA, as shown in Fig. 6 . The pathway targets were marked in white, and the targets of RAB for the treatment of OA were marked in red. The pathway map showed that $\mathrm{RAB}$ was involved in the treatment of $\mathrm{OA}$, including the NF- $\mathrm{KB}$ signalling pathway and PI3K/AKT signalling pathway, with 17 effective targets of RAB in the treatment of OA. It was suggested that $R A B$ may play a role in the treatment of OA by regulating several aspects, and its target may be located in these pathways.

\section{Molecular docking analysis}

Discovery Studio software was used to validate network pharmacology by molecular docking. The LibDock score represents the degree of docking coincidence of molecules. The higher the score is, the better the binding of ligands to receptor proteins is. Molecular docking results showed that RAB had good affinity for the binding of active ingredients to key $\mathrm{OA}$ target protein molecules. Compared with the positive drug celecoxib, RAB had no significant difference in molecular docking fit. At the same time, these findings also indirectly verified that $\mathrm{RAB}$ had a regulatory effect on OA targets, such as CASP3 and MAPK8. The results indicated that the molecular docking results were consistent with the network pharmacology screening results, and the reliability of network pharmacology was verified by molecular docking. The docking results of RAB to the OA protein receptor were shown in Supplementary Table 5. The partial docking process was shown in Fig. 7.

\section{Discussion}

RAB has anti-inflammatory, antitumour and immuneenhancing functions and has been widely used in the treatment of OA [37]. Studies have shown that RAB can delay the progression of $\mathrm{OA}$ and reduce the pathological damage of OA. The drug-containing joint fluid can improve cell viability, promote the proliferation of chondrocytes, increase the expression of type II collagen protein, and reduce the apoptotic rate of chondrocytes, thereby protecting cartilage [32-35]. Although the

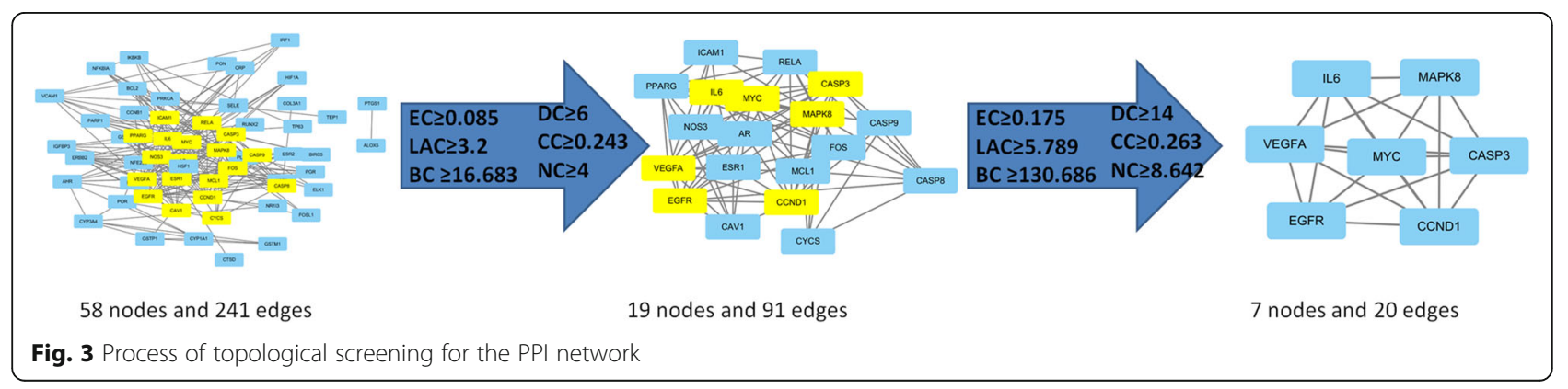


transcriptional activator activity, RNA polymerase II transcription regulatory region sequence-specific DNA bindingnuclear receptor activity transcription factor activity, direct ligand regulated sequence-specific DNA bindingRNA polymerase II proximal promoter sequence-specific DNA bindingproximal promoter sequence-specific DNA bindingprotein heterodimerization activity steroid hormone receptor activity RNA polymerase II transcription factor binding-

$$
\begin{array}{r}
\text { steroid binding } \\
\text { heme binding } \\
\text { integrin binding }
\end{array}
$$
activating transcription factor bindingchromatin bindingtetrapyrrole bindingcofactor binding ubiquitin-like protein ligase binding transcription cofactor bindingHsp90 protein binding-

E-box bindinghistone deacetylase binding -

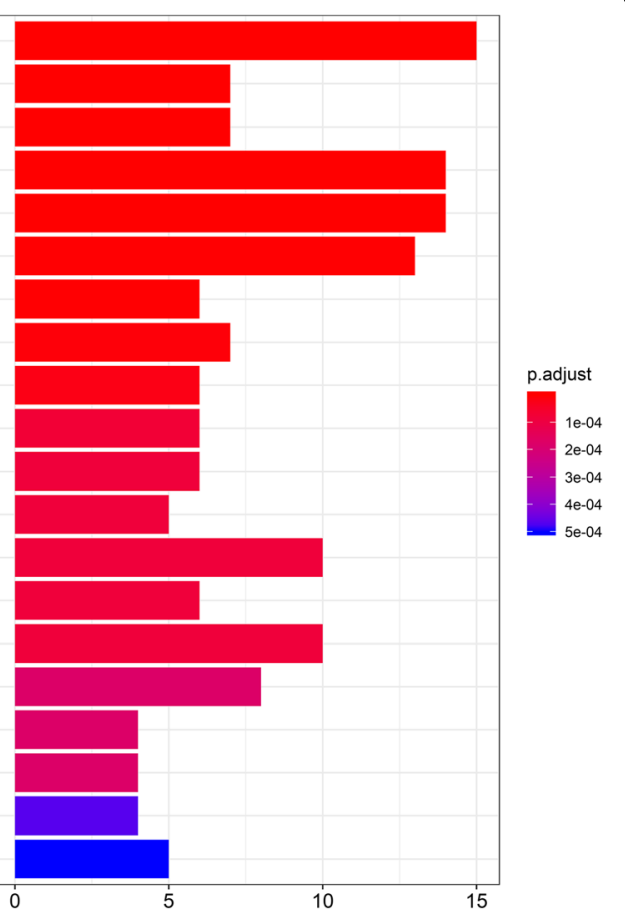

Fig. $4 \mathrm{GO}$ enrichment of Radix Achyranthis Bidentatae active components in the treatment of common targets of osteoarthritis

Kaposi sarcoma-associated herpesvirus infectionAGE-RAGE signaling pathway in diabetic complications Hepatitis BApoptosis -

Fluid shear stress and atherosclerosis Human cytomegalovirus infectionProstate cancer Measles TNF signaling pathwayColorectal cancerIL-17 signaling pathwayEpstein-Barr virus infection-

Proteoglycans in cancerPlatinum drug resistanceApoptosis - multiple species Legionellosis Toxoplasmosis Hepatitis CSmall cell lung cancerPI3K-Akt signaling pathway

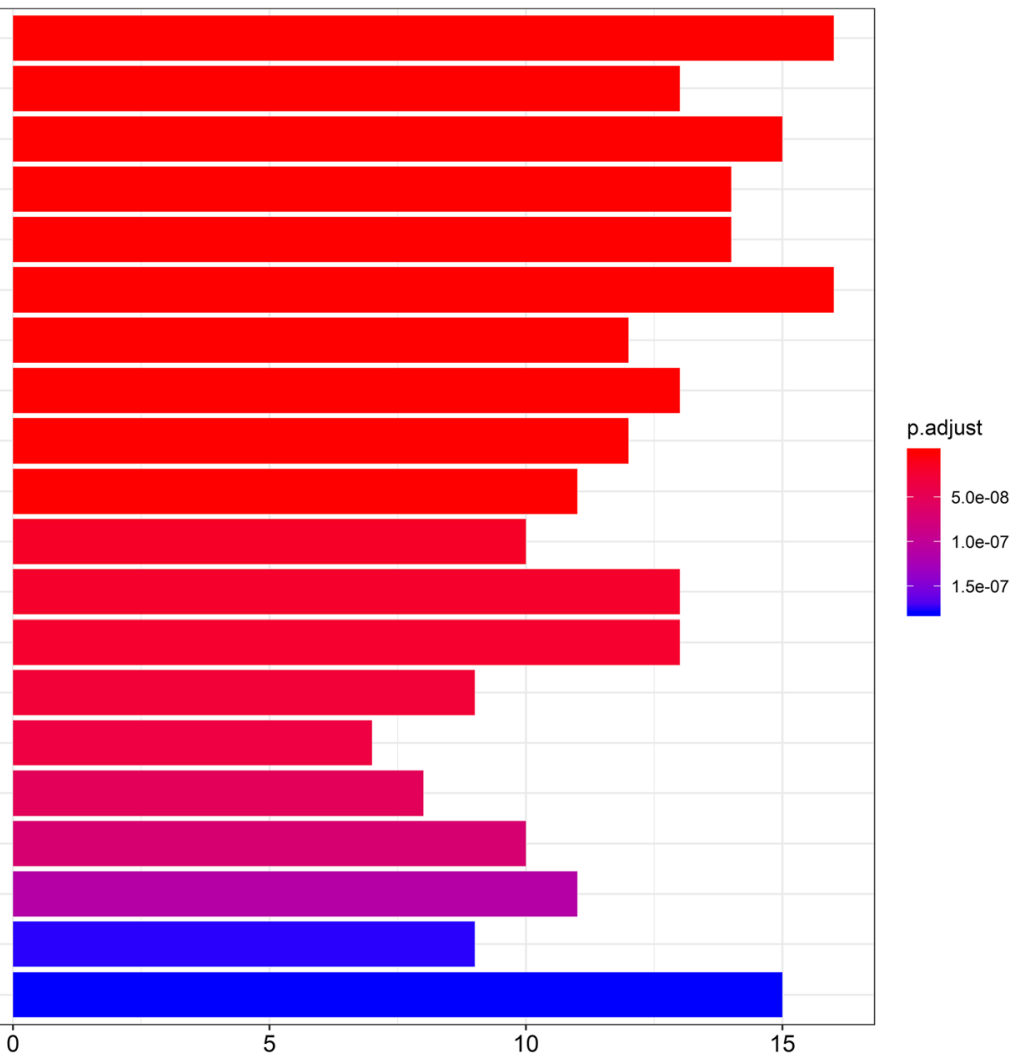

Fig. 5 Enriched KEGG pathways of potential targets for treating osteoarthritis from the main active ingredients of Radix Achyranthis Bidentatae 


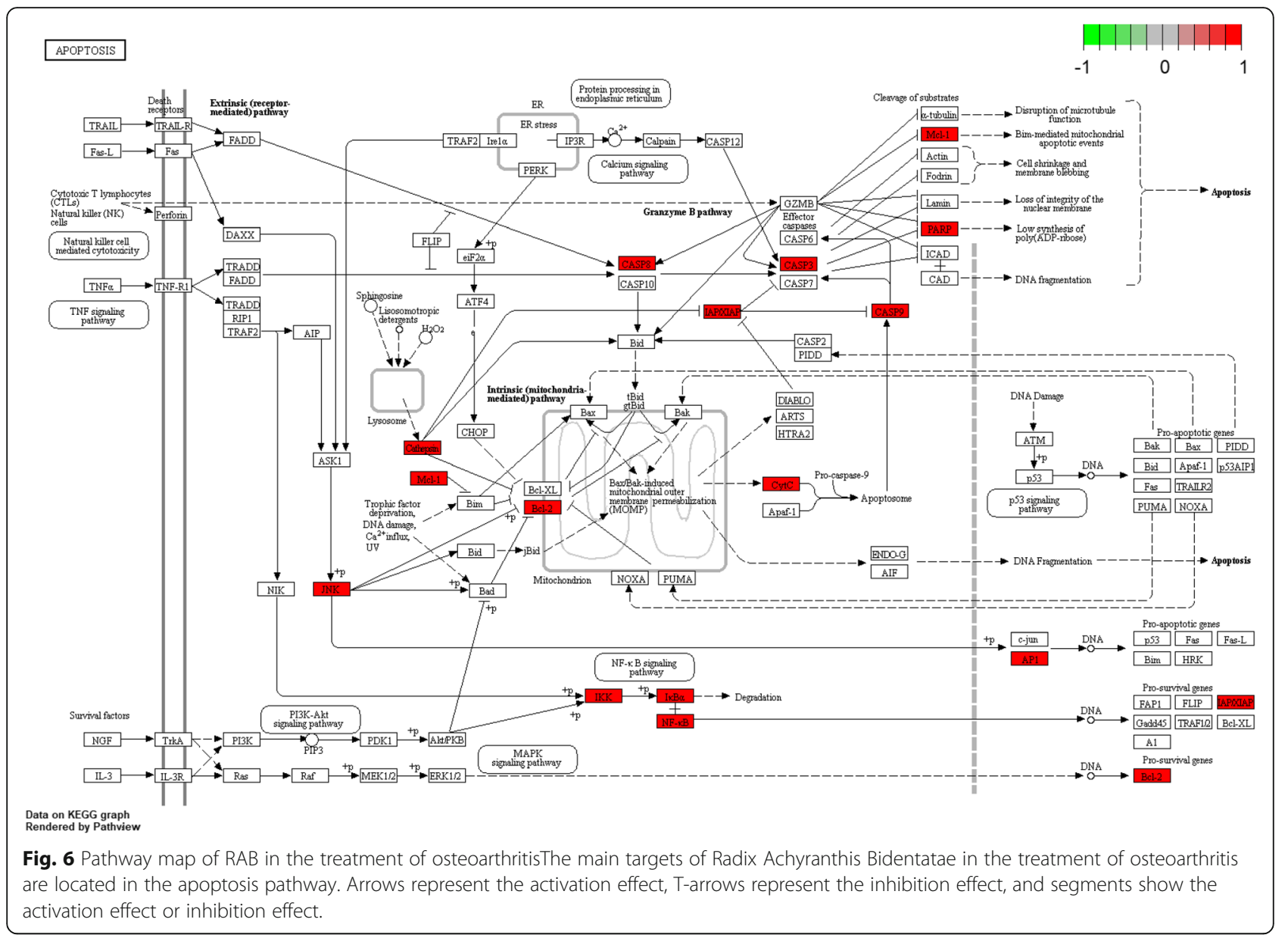

pharmacological effects of the main components of RAB have been studied in depth, the specific mechanism of their action in the treatment of $\mathrm{OA}$ has rarely been reported.

The degree of quercetin, kaempferol, wogonin, betasitosterol, baicalein and stigmasterol was the highest in the CHM-component-target-signalling pathway network. Quercetin inhibits ER stress by activating the sirtuin 1/ adenosine monophosphate activated protein kinase (SIRT1/AMPK) signalling pathway, eliminates knee cartilage degeneration and reduces knee chondrocyte apoptosis. Quercetin also prevents inflammation and proteoglycan degradation by inhibiting the expression of TNF- $\alpha$ and MMP-9 [33]. Kaempferol has an antiinflammatory effect and can reduce the production of inflammatory mediators by reducing the activation of p38 and ERK signalling pathways and inhibiting the NF$\kappa B$ signalling pathway $[38,39]$. Wogonin can inhibit the expression of matrix-degrading protease, enhance the expression of COL2A 1 and ACAN in cartilage, and disturb the balance between ROS and GSH levels. Wogonin also activates $\mathrm{Nrf2}$ by inducing the ROS/ERK/Nrf2/HO-
1-NQO1 signal axis [40]. Baicalein stimulated the expression of anti-apoptotic genes and reduced the production of pro-apoptotic and pro-inflammatory gene products in chondrocytes [40]. In addition, baicalein decreased the expression of protease in vitro and in vivo and the phosphorylation of p38 and ERK but did not decrease the phosphorylation of JNK [32]. Stigmasterol has anti-inflammatory and anti-catabolic properties [41]. Intra-articular injection of stigmasterol could decrease the expression of MMP-1, MMP-3 and MMP-13 in rabbit OA and upregulate the expression of TIMP-1 [31]. Reports on beta-sitosterol are limited. Therefore, quercetin, kaempferol, wogonin, baicalein and stigmasterol may be the most important components in the treatment of OA by RAB.

The top 8 targets of degree in the CHM-componenttarget-signal pathway network were MAPK8, IL-6, EGFR, VEGFA, CCND1, MYC, CASP3, and ESR1. The MAPK8 gene belongs to the MAPK signalling pathway and affects the proliferation and apoptosis of articular chondrocytes. By stimulating the MAPK signalling pathway, pro-inflammatory factors affect the level of MMPs 


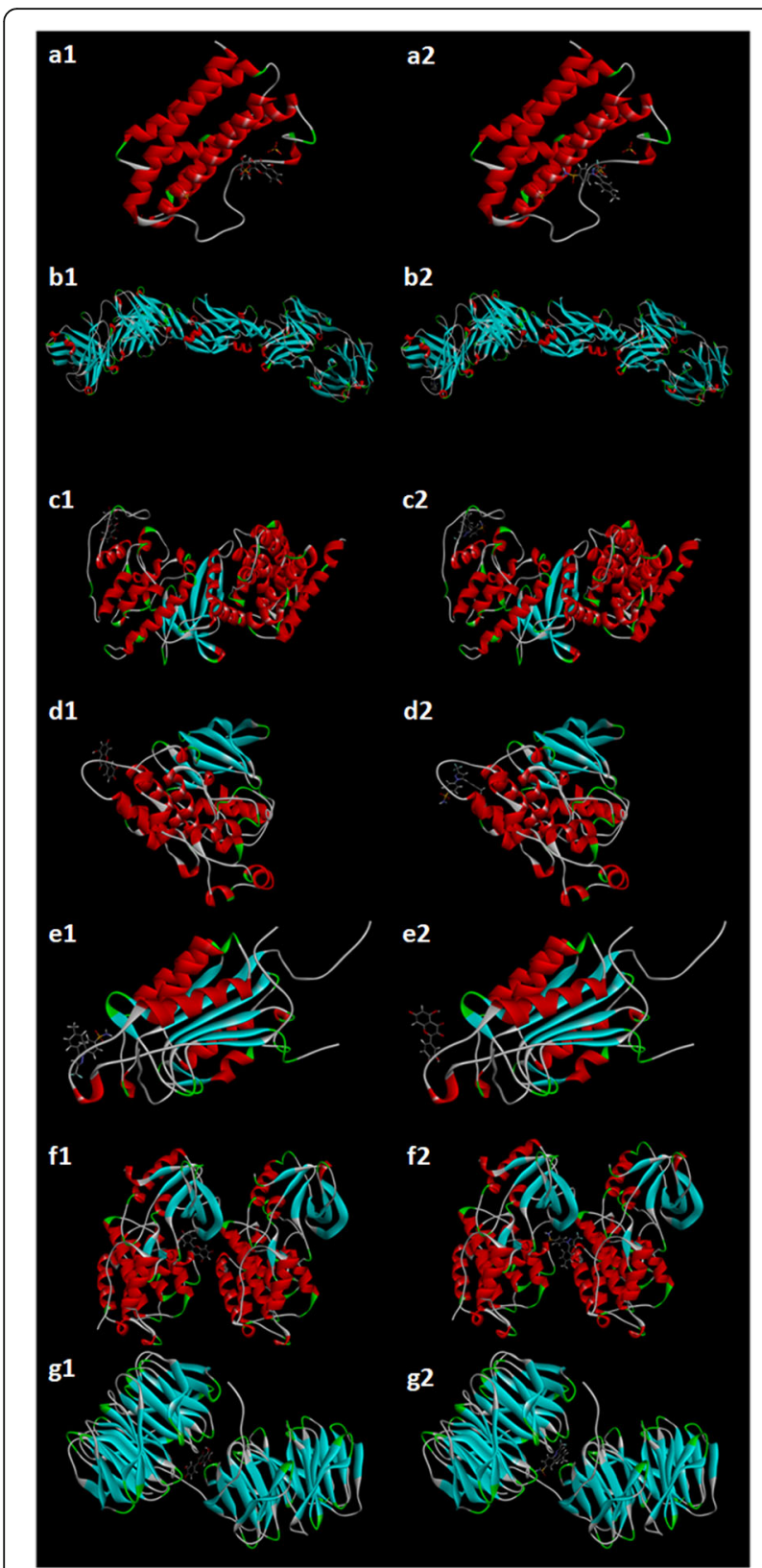

Fig. 7 Molecular docking of compounds with core targets. (a1) Docking process of quercetin with IL6; (a2) Docking process of celecoxib with IL6; (b1) Docking process of quercetin with VEGFA; (b2) Docking process of celecoxib with VEGFA; (c1) Docking process of quercetin with CCND1; (c2) Docking process of celecoxib with CCND1; (d1) Docking process of quercetin with MAPK8; (d2) Docking process of celecoxib with MAPK8; (e1) Docking process of quercetin with CASP3; (e2) Docking process of celecoxib with CASP3; (f1) Docking process of quercetin with EGFR; (f2) Docking process of celecoxib with EGFR; (g1) Docking process of quercetin with MYC; (g2) Docking process of celecoxib with MYC

and promote the development of OA [42]. IL-6 is a cytokine that strongly activates the immune system and enhances the inflammatory response. Recent studies have shown that IL-6 induces chondrocyte catabolism primarily through STAT3 signalling, and by blocking IL6 or STAT3, it can alleviate the symptoms of OA mice caused by medial meniscus instability [43]. EGFR is highly expressed in healthy articular cartilage, promotes the secretion of lubricants in articular cartilage, and plays an important role in cell growth, proliferation and differentiation. The expression of the VEGFA gene was associated with early degenerative changes of cartilage and subchondral bone [44]. Studies have shown that synovial vascular endothelial growth factor may be involved in the pain pathway of KOA and may be mediated by apelin [45]. Overexpression of CCND1 reversed the effect of FOXD2-AS1 inhibition on chondrocyte viability [46]. Related studies have shown that Cyclin D1 (a protein encoded by the human CCND1 gene) silencing inhibits IL-1 $\beta$-induced proliferation of rat chondrocytes induced by OA and induces apoptosis [47]. The MYC gene is a group of previously characterized oncogenes, including C-myc, $\mathrm{N}$-myc, and L-myc. A study found that $\mathrm{C}$-myc gene silencing promotes cell proliferation and inhibits IL-1 $\beta$-induced rat chondrocyte apoptosis and cytokine expression [48]. CASP3 was determined to play an important role in the apoptosis of articular chondrocytes in KOA rats. The expression of CASP3 is positively correlated with the apoptosis of articular chondrocytes [49]. The ESR1 gene can be expressed in osteoblasts, osteoclasts, chondrocytes and bone marrow stromal cells, as well as bone cells. E2 targeting activation of ESR1 in chondrocytes can inhibit ERK phosphorylation activation, thereby inhibiting the ERK signalling pathway, promoting the formation of autophagic flow, activating autophagy, reducing apoptosis, and causing chondrocyte proliferation [50].

The results of enrichment analysis showed that the main pathways involved in the treatment process are cell apoptosis, the PI3K/AKT pathway, the IL-17 signalling pathway, and viral infection. Apoptosis is the programmed death of cells. Modern studies have found that the signalling pathways that regulate the apoptosis of OA chondrocytes are complex and diverse. Qin et al found that specific inhibition of the SDF-1/CXCR4 signalling pathway can regulate subchondral bone microstructure and attenuate OA pathological damage [51]. Kalaitzoglouë observed that the activation of the TLR4 signalling pathway induces cartilage catabolism in middle-aged female mice, thereby aggravating the progression of OA [52]. MAPK is a serine/threonine protein kinase in cells. As one of the important signal transduction pathways in eukaryotic cells, MAPK participates in the proliferation, differentiation and apoptosis of chondrocytes, induces the secretion of matrix metalloproteinases and causes cartilage matrix degradation. The abnormal expression of MAPK can accelerate the process of OA [53]. 
The PI3K/AKT pathway is an important antiapoptotic signalling pathway in vivo that can promote the apoptosis and autophagy of chondrocytes and play an important role in the development of OA [54, 55]. AKT, a serine/threonine protein kinase, is activated by PI3K and recruited to the plasma membrane, which plays an important role in regulating cell growth and apoptosis. Recent studies have shown that AKT activation can regulate autophagy and improve cartilage injury and related indicators.

IL-17 plays an important role in OA. Accurate and effective regulation of IL-17 signal transduction can prevent inflammation. A large number of studies have shown that IL-17 is overexpressed in the synovium of RA patients, but there are few studies on the expression of IL-17 in OA patients and its relationship with the severity of arthritis [56-58]. Some scholars have found that the level of IL-17 is related to the severity of knee pain in osteoarthritis. Blocking the IL-17 signalling pathway can delay the pain associated with osteoarthritis [59]. A meta-analysis also showed that the pathogenesis of KOA in the Chinese Han population may be positively correlated with IL-17A (rs2275913) [60].

This study has a number of limitations. There are notably few studies investigating the relationship between viral pathways and OA. These results may be observed because the current research data on virusrelated pathways are more abundant, and the analysis in the existing database will produce certain bias. Therefore, virus-related pathways, such as Kaposi's sarcoma-associated herpesvirus infection, hepatitis B, hepatitis $\mathrm{C}$, human cytomegalovirus infection and other viral-related pathways, can also be involved in the treatment of OA.

In summary, this study used network pharmacological methods and techniques to identify 17 kinds of drug components and 101 potential targets in RAB. There were 2515 OA disease-related targets, 63 of which were RAB. Through enrichment analysis of GO biological processes and KEGG signalling pathways, it was preliminarily predicted that $\mathrm{RAB}$ may regulate the targets of MAPK8, IL-6, VEGFA, CCND1, CASP3, and ESR1 through quercetin, kaempferol, wogonin, betasitosterol, baicalein, and stigmasterol. The regulation of apoptosis signalling pathways, IL-17 signalling pathways, and PI3K/AKT signalling pathways ultimately inhibit the inflammatory response, regulate immune function and regulate cell apoptosis to treat OA. Due to the limitations of database data and corresponding analysis algorithms and software functions of various platforms, confirming these results requires experimental research, and the specific mechanism governing these phenomena also needs to be elucidated by experimental research.

\section{Conclusions}

This study investigated the effective active ingredients and molecular mechanisms of RAB in the treatment of OA from the perspective of network pharmacology. The active ingredients of $\mathrm{RAB}$ in $\mathrm{OA}$ treatment are composed of 16 compounds; among them, quercetin, kaempferol, wogonin, beta-sitosterol, baicalein and stigmasterol are the important active ingredients. There are 63 target genes involved in the treatment of $\mathrm{OA}$ by $\mathrm{RAB}$, among which MAPK8, IL-6, EGFR, VEGFA, CCND1, MYC, CASP3 and ESR1 are the key target genes. The signalling pathways of RAB in the treatment of OA mainly include the apoptosis signalling pathway, the IL-17 signalling pathway and the PI3K/AKT signalling pathway. In addition, the results of this study provide a new way to further study the mechanism of RAB in the treatment of OA.

\section{Supplementary information}

Supplementary information accompanies this paper at https://doi.org/10. 1186/s12906-020-02909-4.

Additional file 1. Supplementary Table 1: Potential target information of RAB for $O A$.

Additional file 2. Supplementary Table 2: Active ingredient parameters of RAB.

Additional file 3. Supplementary Table 3: Common gene of RAB in the treatment of $\mathrm{OA}$.

Additional file 4. Supplementary Table 4: Compound-target pair information.

Additional file 5. Supplementary Table 5: Results of ligand-receptor protein molecular docking.

\section{Abbreviations}

RAB: Radix achyranthis bidentatae; CHM: Chinese herbal medicine; OA: Osteoarthritis; TCMSP: traditional Chinese medicine systems pharmacology; OB: Oral bioavailability; DL: Drug-likeness; KEGG: Kyoto encyclopedia of genes and gnomes; PDB ID: Protein data bank identification; PPI network: Protein-protein interaction network; MF: Molecular function; BP: Biological process; CC: Cellular component

\section{Acknowledgements}

We gratefully acknowledge the contributions of Chenghua Zhang and Junyao Xu (Nanjing University of Traditional Chinese Medicine) in the study design and data analysis.

\section{Declarations}

All declarations in the manuscript are as follows:

\section{Authors' contributions}

ZLG and SXQ contributed equally to this paper, and they designed the study. Experimental work and data collection were conducted by SXQ and XRL. HZQ and SXQ analysed and interpreted the data. SXQ and ZLG drafted the manuscript. WPM and MJ and ZLG provided critical comments and revised the manuscript. MW and DL and $Z \mathrm{~L}$ helped perform the analysis with constructive discussions. All authors read and approved the final version of the manuscript.

\section{Funding}

This study was supported by the project Leading Talents of Traditional Chinese Medicine in Jiangsu Province (SLJ0207). The present study was supported by the National Natural Science Foundation of China (81774334) and the Jiangsu Provincial Science and Technology Department Social 
Development Fund (BE2017774). The funders had no role in study design, data collection and analysis, decision to publish, or preparation of the manuscript.

\section{Availability of data and materials}

The datasets used and/or analysed during the current study are available from the corresponding author on reasonable request.

\section{Ethics approval and consent to participate}

Not applicable.

\section{Consent for publication}

Not applicable.

\section{Competing interests}

All authors declare there are no competing interests.

\section{Received: 25 October 2019 Accepted: 31 March 2020}

Published online: 21 April 2020

\section{References}

1. Sun X, Zhen X, Hu X, Li Y, Gu S, Gu Y, Dong H. Osteoarthritis in the MiddleAged and Elderly in China: Prevalence and Influencing Factors. Int J Environ Res Public Health. 2019;16(23). PubMed PMID: 31779104; PubMed Central PMCID: PMC6926632. https://doi.org/10.3390/ijerph16234701. https://www. mdpi.com/1660-4601/16/23/4701.

2. Jordan KP, Joud A, Bergknut C, Croft P, Edwards JJ, Peat G, Petersson IF, Turkiewicz A, Wilkie R, Englund M. International comparisons of the consultation prevalence of musculoskeletal conditions using populationbased healthcare data from England and Sweden. Ann Rheum Dis. 2014; 73(1):212-8

3. Peat G, McCarney R, Croft P. Knee pain and osteoarthritis in older adults: a review of community burden and current use of primary health care. Ann Rheum Dis. 2001:60(2):91-7.

4. GBD 2016 Stroke Collaborators. Global, regional, and national burden of stroke, 1990-2016: a systematic analysis for the Global Burden of Disease Study 2016. Lancet Neurol. 2019;18(5):439-58. PubMed PMID: 30871944; PubMed Central PMCID: PMC6494974. https://doi.org/10.1016/S14744422(19)30034-1. Epub 2019 Mar 11.

5. Xu BP, Yao M, Tian ZR, Zhou LY, Yang L, Li ZJ, Zhu S, Wang XT, Lan JH, Wang YJ, Cui XJ. Study on efficacy and safety of Tong-luo Qu-tong plaster treatment for knee osteoarthritis: study protocol for a randomized, doubleblind, parallel positive controlled, multi-center clinical trial. Trials. 2019;20(1): 377.

6. Chen FP, Chang CM, Hwang SJ, Chen YC, Chen FJ. Chinese herbal prescriptions for osteoarthritis in Taiwan: analysis of National Health Insurance dataset. BMC Complement Altern Med. 2014;14:91.

7. Zhang W, Wang S, Zhang R, Zhang Y, Li X, Lin Y, Wei X. Evidence of Chinese herbal medicine Duhuo Jisheng decoction for knee osteoarthritis: a systematic review of randomised clinical trials. BMJ Open. 2016;6:e008973.

8. Wang F, Shi L, Zhang Y, Wang K, Pei F, Zhu H, Shi Z, Tao T, Li Z, Zeng P, Wang X, Ji Q, Qin L, Xue Q. A traditional herbal formula Xianlinggubao for pain control and function improvement in patients with knee and hand osteoarthritis: a multicenter, randomized, open-label, Controlled Trial. Evid Based Complement Alternat Med. 2018;2018:1827528.

9. Peici H. Data Mining and Clinical Drug Efficacy Analysis of Traditional Chinese Medicine in the Treatment of Knee Osteoarthritis. doctor: Nanjing University of Chinese Medicine; 2015.

10. Jianke $P$, Kuihao $H$, Jun $L$, Jiyuan $Y$, Yongming $H$, Minghui L. Study on drug use of knee osteoarthritis based on association rules and complex system entropy clustering. Chin J Exp Tradit Med Formulae. 2015;21(12):201-4.

11. Yang $L$, Jiang $H$, Yan $M L$, Xing $X D$, Zhang $Y Y$, Wei $N$, Yang $B Y, W a n g ~ Q H$, Kuang HX. A new phytoecdysteroid from the roots of Achyranthes bidentata Bl. Nat Prod Res. 2017;31(9):1073-9.

12. He X, Wang X, Fang J, Chang Y, Ning N, Guo H, Huang L, Huang X. The genus Achyranthes: a review on traditional uses, phytochemistry, and pharmacological activities. J Ethnopharmacol. 2017;203:260-78.

13. Shang FQ. Comparative study on Achvranthese hidentata B1 and Cvathula officinalis Kuan. [D] The Wuhan Botanical Garden of the Chinese Academy of Sciences, 2016.
14. He G, Guo W, Lou Z, Zhang H. Achyranthes bidentata saponins promote osteogenic differentiation of bone marrow stromal cells through the ERK MAPK signaling pathway. Cell Biochem Biophys. 2014;70(1):467-73.

15. $X u X X$, Zhang $X H$, Diao $Y$, Huang $Y X$. Achyranthes bidentate saponins protect rat articular chondrocytes against interleukin-1 beta-induced inflammation and apoptosis in vitro. Kaohsiung J Med Sci. 2017;33(2): 62-8.

16. Weng $X$, Lin P, Liu F, Chen J, Li H, Huang L, Zhen C, Xu H, Liu X, Ye H, et al. Achyranthes bidentata polysaccharides activate the Wnt/beta-catenin signaling pathway to promote chondrocyte proliferation. Int J Mol Med. 2014;34(4):1045-50.

17. Song D, Cao Z, Huang S, Tickner J, Li N, Qiu H, Chen X, Wang C, Chen K, Sun $Y$, et al. Achyranthes bidentata polysaccharide suppresses osteoclastogenesis and bone resorption via inhibiting RANKL signaling. J Cell Biochem. 2018;119(6):4826-35.

18. Hua S, Zhang X. Effects of Achyranthes bidentata alcohol on proliferation capacity of osteoblasts and miRNA in Runx2. Exp Ther Med. 2019;18(3): $1545-50$.

19. Li S, Zhang ZQ, Wu LJ, Zhang XG, Li YD, Wang YY. Understanding ZHENG in traditional Chinese medicine in the context of neuro-endocrine-immune network. IET Syst Biol. 2007;1(1):51-60.

20. Ru J, Li P, Wang J, Zhou W, Li B, Huang C, Li P, Guo Z, Tao W, Yang Y, et al. TCMSP: a database of systems pharmacology for drug discovery from herbal medicines. J Cheminform. 2014;6:13.

21. Wan $Y, X u L$, Liu Z, Yang M, Jiang $X$, Zhang Q, Huang J. Utilising network pharmacology to explore the underlying mechanism of Wumei pill in treating pancreatic neoplasms. BMC Complement Altern Med. 2019;19(1): 158.

22. Xu X, Zhang W, Huang C, Li Y, Yu H, Wang Y, Duan J, Ling Y. A novel chemometric method for the prediction of human oral bioavailability. Int J Mol Sci. 2012;13(6):6964-82.

23. Shannon P, Markiel A, Ozier O, Baliga NS, Wang JT, Ramage D, Amin N, Schwikowski B, Ideker T. Cytoscape: a software environment for integrated models of biomolecular interaction networks. Genome Res. 2003;13(11): 2498-504.

24. Zhou WX, Wang TX, Cheng XR, Han L, Zhang YX. Techniques of molecular network analysis in study on network pharmacology. J Int Pharmaceutical Res. 2016;43(03):399-409.

25. Hopkins AL. Network pharmacology: the next paradigm in drug discovery. Nat Chem Biol. 2008:4(11):682-90

26. Yu H, Kim PM, Sprecher E, Trifonov V, Gerstein M. The importance of bottlenecks in protein networks: correlation with gene essentiality and expression dynamics. PLoS Comput Biol. 2007;3(4):e59.

27. Athanasios A, Charalampos V, Vasileios T, Ashraf GM. Protein-protein interaction (PPI) network: recent advances in drug discovery. Curr Drug Metab. 2017;18(1):5-10.

28. Tang Y, Li M, Wang J, Pan Y, Wu FX. CytoNCA: a cytoscape plugin for centrality analysis and evaluation of protein interaction networks. Biosystems. 2015;127:67-72.

29. Li X, He P, Hou Y, Chen S, Xiao Z, Zhan J, Luo D, Gu M, Lin D. Berberine inhibits the interleukin-1 beta-induced inflammatory response via MAPK downregulation in rat articular chondrocytes. Drug Dev Res. 2019;80(5):63745. PubMed PMID: 31032997. Epub 2019 Apr 29. https://doi.org/10.1002/ddr. 21541. Epub 2019 Apr 29.

30. Wong SK, Chin KY, Ima-Nirwana S. Berberine and musculoskeletal disorders: The therapeutic potential and underlying molecular mechanisms. Phytomedicine. 2019. p. 152892. Review. PubMed PMID: 30902523. https:// doi.org/10.1016/j.phymed.2019.152892. [Epub ahead of print].

31. Gabay O, Sanchez C, Salvat C, Chevy F, Breton M, Nourissat G, Wolf C, Jacques C, Berenbaum F. Stigmasterol: a phytosterol with potential antiosteoarthritic properties. Osteoarthr Cartil. 2010;18(1):106-16.

32. Chen WP, Xiong Y, Hu PF, Bao JP, Wu LD. Baicalein inhibits MMPs expression via a MAPK-dependent mechanism in chondrocytes. Cell Physiol Biochem. 2015;36(1):325-33.

33. Feng $K$, Chen Z, Pengcheng $L$, Zhang S, Wang X. Quercetin attenuates oxidative stress-induced apoptosis via SIRT1/AMPK-mediated inhibition of ER stress in rat chondrocytes and prevents the progression of osteoarthritis in a rat model. J Cell Physiol. 2019;234(10):18192-205.

34. Wei B, Zhang Y, Tang L, Ji Y, Yan C, Zhang X. Protective effects of quercetin against inflammation and oxidative stress in a rabbit model of knee osteoarthritis. Drug Dev Res. 2019;80(3):360-7. 
35. Qiu L, Luo Y, Chen X. Quercetin attenuates mitochondrial dysfunction and biogenesis via upregulated AMPK/SIRT1 signaling pathway in OA rats. Biomed Pharmacother. 2018;103:1585-91.

36. Li Y, Wang J, Song X, Bai H, Ma T, Zhang Z, Li X, Jiang R, Wang G, Fan X, et al. Effects of baicalein on IL-1beta-induced inflammation and apoptosis in rat articular chondrocytes. Oncotarget. 2017:8(53):90781-95.

37. Chongjing S, Shanshan W, Xiaomei L, Zhongqin C, Qianghua Y, Ying S. Discussion on the current situation of Achyranthes bidentata. Asia-Pac Traditional Med. 2017;13(22):55-8.

38. Zhuang Z, Ye G, Huang B. Kaempferol Alleviates the Interleukin-1ß-Induced Inflammation in Rat Osteoarthritis Chondrocytes via Suppression of NF-KB. Med Sci Monit. 2017;23:3925-931. PubMed PMID: 28806392; PubMed Central PMCID: PMC5566200.

39. Huang X, Pan Q, Mao Z, Wang P, Zhang R, Ma X, Chen J, You H. Kaempferol inhibits interleukin 1 beta stimulated matrix metalloproteinases by suppressing the MAPKassociated ERK and P38 signaling pathways. Mol Med Rep. 2018;18(3):2697-704.

40. Khan NM, Haseeb A, Ansari MY, Devarapalli P, Haynie S, Haqqi TM. Wogonin, a plant derived small molecule, exerts potent anti-inflammatory and chondroprotective effects through the activation of ROS/ERK/Nrf2 signaling pathways in human osteoarthritis chondrocytes. Free Radic Biol Med. 2017;106:288-301.

41. Chen WP, Yu C, Hu PF, Bao JP, Tang JL, Wu LD. Stigmasterol blocks cartilage degradation in rabbit model of osteoarthritis. Acta Biochim Pol. 2012;59(4): 537-41.

42. Sufang F, Wenya W, Lv Z. Effect of mitogen-activated protein kinase signaling pathway on osteoarthritis cartilage. Chinese J Tissue Eng Res. 2012;16(37):7015-9.

43. Latourte A, Cherifi C, Maillet J, Ea HK, Bouaziz W, Funck-Brentano T, CohenSolal M, Hay E, Richette P. Systemic inhibition of IL-6/Stat3 signalling protects against experimental osteoarthritis. Ann Rheum Dis. 2017;76(4): 2016-209757

44. Zupan J, Vrtačnik P, Cör A, Haring G, Weryha G, Visvikis-Siest S, Marc J. VEGF-A is associated with early degenerative changes in cartilage and subchondral bone. Growth Factors. 2018;36(5-6):263-273. PubMed PMID: 30764675. https://doi.org/10.1080/08977194.2019.1570926. Epub 2019 Feb 15

45. Takano S, Uchida K, Inoue G, Matsumoto T, Aikawa J, Iwase D, Mukai M, Miyagi M, Takaso M. Vascular endothelial growth factor expression and their action in the synovial membranes of patients with painful knee osteoarthritis. BMC Musculoskelet Disord. 2018;19(1):204.

46. Cao L, Wang Y, Wang Q, Huang J. LncRNA FOXD2-AS1 regulates chondrocyte proliferation in osteoarthritis by acting as a sponge of miR206 to modulate CCND1 expression. Biomed Pharmacother. 2018;106:1220 26. PubMed PMID: 30119190. https://doi.org/10.1016/j.biopha.2018.07.048. Epub 2018 Jul 19.

47. Zan PF, Yao J, Wu Z, Yang Y, Hu S, Li GD. Cyclin D1 Gene Silencing Promotes IL-1 $\beta$-Induced Apoptosis in Rat Chondrocytes. J Cell Biochem. 2018;119(1):290-99. Epub 2017 Jul 4. PubMed PMID: 28548679. https://doi. org/10.1002/jcb.26172. https://onlinelibrary.wiley.com/doi/abs/10.1002/ jcb.26172.

48. Zou J, Li XL, Shi ZM, Xue JF. Effects of C - myc gene silencing on interleukin-1 $\beta$-induced rat chondrocyte cell proliferation, apoptosis and cytokine expression. J Bone Miner Metab. 2017;36(3):1-11.

49. Jinming $S$, Lei F, Jie C, Yu W, Jiannong Y. Experimental study on Simiaosan regulating chondrocyte apoptosis and autophagy in the treatment of knee osteoarthritis in rats. J New Chinese Med. 2017;49(09):12-5.

50. Min L, Weiwei X, Wei Z, Danyang Y, Rui L, Fengjin G. Targeted binding of estradiol and ESR1 regulates chondrocyte proliferation through ERK signaling pathway. J Southern Med Univ. 2019;39(02):134-43.

51. Qin HJ, Xu T, Wu HT, Yao ZL, Hou YL, Xie YH, Su JW, Cheng CY, Yang KF, Zhang XR, et al. SDF-1/CXCR4 axis coordinates crosstalk between subchondral bone and articular cartilage in osteoarthritis pathogenesis. BONE. 2019:125:140-50.

52. Kalaitzoglou E, Lopes E, Fu Y, Herron JC, Flaming JM, Donovan EL, Hu Y, Filiberti A, Griffin TM, Humphrey MB. TLR4 promotes and DAP12 limits obesity-induced osteoarthritis in aged female mice. JBMR Plus. 2019;3(4) e10079.

53. Huang X, Xi Y, Mao Z, Chu X, Zhang R, Ma X, Ni B, Cheng H, You H. Vanillic acid attenuates cartilage degeneration by regulating the MAPK and PI3K AKT/NF-kappaB pathways. Eur J Pharmacol. 2019;859:172481.
54. Xue JF, Shi ZM, Zou J, Li XL. Inhibition of PI3K/AKT/mTOR signaling pathway promotes autophagy of articular chondrocytes and attenuates inflammatory response in rats with osteoarthritis. Biomed Pharmacother. 2017:89:1252-61.

55. Xiaoyan L, Yan Z, Chunping D, Guifu W. Study on the correlation between PI3K AKT signaling pathway function and apoptosis in cartilage tissue of osteoarthritis rat model. J Hainan Med Univ. 2017;23(11):1452-5.

56. Roeleveld DM, Koenders MI. The role of the Th17 cytokines IL-17 and IL-22 in rheumatoid arthritis pathogenesis and developments in cytokine immunotherapy. CYTOKINE. 2015;74(1):101-7.

57. Zheng $Y$, Sun $L$, Jiang T, Zhang D, He D, Nie H. TNFalpha promotes Th17 cell differentiation through IL-6 and IL-1beta produced by monocytes in rheumatoid arthritis. J Immunol Res. 2014;2014:385352.

58. van Baarsen LG, Lebre MC, van der Coelen D, Aarrass S, Tang MW, Ramwadhdoebe TH, Gerlag DM, Tak PP. Heterogeneous expression pattern of interleukin 17A (IL-17A), IL-17F and their receptors in synovium of rheumatoid arthritis, psoriatic arthritis and osteoarthritis: possible explanation for nonresponse to anti-IL-17 therapy? Arthritis Res Ther. 2014 16(4):426.

59. Liu Y, Peng H, Meng Z, Wei M. Correlation of IL-17 level in synovia and severity of knee osteoarthritis. Med Sci Monit. 2015;21:1732-6.

60. Zhang PL, Yang FM, Qiao ZZ, Liu J, Yang QY, Wang YJ, Qi M, Cui LN, Meng L, Li XP. Association between interleukin-17A and 17F single nucleotide polymorphisms and knee osteoarthritis. Zhonghua Yi Xue Za Zhi. 2019; 99(24):1870-4.

\section{Publisher's Note}

Springer Nature remains neutral with regard to jurisdictional claims in published maps and institutional affiliations.
Ready to submit your research? Choose BMC and benefit from:

- fast, convenient online submission

- thorough peer review by experienced researchers in your field

- rapid publication on acceptance

- support for research data, including large and complex data types

- gold Open Access which fosters wider collaboration and increased citations

- maximum visibility for your research: over $100 \mathrm{M}$ website views per year

At $\mathrm{BMC}$, research is always in progress.

Learn more biomedcentral.com/submissions 